DOI: 10.24835/1607-0763-2018-4-85-95

Комплексная лучевая диагностика

у наркозависимых пациентов с фосфорными

остеонекрозами лицевого скелета

на дооперационном этапе

\author{
Бабкова А.А. ${ }^{1 *}$, Серова Н.С. ${ }^{1}$, Медведев Ю.А. ${ }^{2}$, Паша С.П. ${ }^{1}$ \\ 1 ФГАОУ ВО Первый МГМУ им. И.М. Сеченова Минздрава России (Сеченовский Университет), Университетская \\ клиническая больница №1, Москва, Россия \\ 2 ФГБОУ ВО МГМСУ им. А.И. Евдокимова Минздрава России, Клинический медицинский центр, Москва, Россия
}

\title{
Complex radialogical diagnostics in drug-dependent patients with phosphorus osteonecrosis of the facial skeleton at the preoperative stage
}

\author{
Babkova A.A. ${ }^{1 *}$, Serova N.S. ${ }^{1}$, Medvedev Yu.A. ${ }^{2}$, Pasha S.P. ${ }^{1}$ \\ 1 I.M. Sechenov First Moscow State Medical University, University Hospital № 1, Moscow, Russia \\ ${ }^{2}$ A.I. Yevdokimov Moscow State University of Medicine and Dentistry, Clinical medical center, Moscow, Russia
}

\begin{abstract}
Цель исследования: определить роль комплексной лучевой диагностики и диагностическую эффективность различных лучевых методов у наркозависимых пациентов с фосфорными остеонекрозами лицевого скелета на дооперационном этапе.

Материал и методы. В представленное исследование было включено 85 наркозависимых пациентов с диагнозом "токсический фосфорный остеонекроз" лицевого скелета. В предоперационном периоде всем пациентам было выполнено полное клинико-лучевое обследование. Ортопантомография (ОПТГ), рентгенография черепа, мультиспиральная компьютерная томография (МСКТ), конусно-лучевая компьютерная томография (КЛКТ) были проведены 85 (100\%) пациентам на дооперационном этапе. Также 19 (22,4\%) пациентам была проведена радионуклидная диагностика, включившая в себя планарную сцинтиграфию и однофотонноую эмиссионную компьютерную томографию (ОФЭКТ). Далее всем пациентам было проведено хирургическое лечение в объеме полной или частичной резекции челюстей и других костей лицевого скелета.
\end{abstract}

Результаты. Данные МСКТ и КЛКТ позволили выявить распространение патологического процесса на другие кости лицевого (в ряде случаев мозгового) отдела скелета у 25 (29,5\%) пациентов, данные радионуклидной диагностики - зоны накопления радио- фармпрепарата как в костях черепа, так и за его пределами. По результатам анализа полученных данных была определена роль комплексной лучевой диагностики, а также диагностическая эффективность лучевых методов (ОПТГ, рентгенографии черепа, МСКТ, КЛКТ) в обследовании наркозависимых пациентов с фосфорными остеонекрозами лицевого скелета на дооперационном этапе.

Заключение. Комплексная лучевая диагностика с применением высокоинформативных современных методов (МСКТ, КЛКТ, радионуклидная диагностика) является неотъемлемым этапом обследования пациентов с остеонекрозами лицевого скелета на дооперационном этапе. Показатели диагностической эффективности для МСКТ составили: чувствительность - 98,1\%, специфичность - 99,6\%, точность - 98,8\%, для КЛКТ: чувствительность - 97,3\%, специфичность - 99,1\%, точность - 98,4\%, для ОПТГ: чувствительность - 78,6\%, специфичность - 76,3\%, точность - 77,4\%, для рентгенографии черепа: чувствительность - $61,1 \%$, специфичность - 59,2\%, точность - 60,2\%.

Ключевые слова: остеонекроз, мультиспиральная компьютерная томография, ортопантомография, дезоморфин, конусно-лучевая компьютерная томография, радионуклидная диагностика. 
Ссылка для цитирования: Бабкова А.А., Серова Н.С., Медведев Ю.А., Паша С.П. Комплексная лучевая диагностика у наркозависимых пациентов с фосфорными остеонекрозами лицевого скелета на дооперационном этапе. Медицинская визуализация. 2018; 22 (4): 85-95. DOI: 10.24835/1607-0763-2018-4-85-95.

$$
\star \star \star
$$

The purpose. To determine the role of complex radiological diagnostics and diagnostic effectiveness of various radiological methods in drug-dependent patients with phosphorous osteonecrosis of the facial skeleton at the preoperative stage.

Materials and methods. The study included 85 drug addicted patients with a diagnosis of toxic phosphorus osteonecrosis of the facial skeleton. In the preoperative period all patients underwent full clinical and radiological examination. Orthopantomography (OPG), skull x-ray, multislice computed tomography (MSCT), cone-beam computed tomography (CBCT) were performed in 85 patients (100\%) at the preoperative stage. Also, 19 patients (22.4\%) underwent radionuclide diagnostics, which included planar scintigraphy and single-photon emission computed tomography (SPECT). Further, all patients underwent surgical treatment to the extent of complete or partial resection of the jaws and other bones of the facial skeleton.

Results. According to the data of complex radiological examination at the preoperative stage it was revealed that in $48 \%(n=41)$ cases the osteonecrosis zones were localized in the lower jaw, in 24\% $(n=20)$ in the upper jaw, in 28\% (24) patients the pathological process captured both jaws. Also, MSCT and CBCT data revealed the spread of the pathological process to other bones of the facial, in some cases, the brain part of the scull in 25 patients $(29.5 \%)$. The data of radionuclide diagnostics allowed to reveal the zones of radiopharmaceutical accumulation both in the bones of the skull and outside it. Based on the results of the analysis of the data obtained, the role of complex radiation diagnosis was determined, as well as the diagnostic effectiveness of radiation methods (OPG, X-ray of the skull, MSCT, CBCT) in the examination of drug-dependent patients with phosphoric osteonecrosis of the facial skeleton at the preoperative stage.

Conclusion. The complex radiological diagnostics with application of high informative modern methods (MSCT, $\mathrm{CBCT}$, radionuclide diagnostics) is the required step in the evaluation of patients with osteonecrosis of the facial skeleton at the preoperative stage. The diagnostic efficiency parameters for MSCT were: sensitivity - 98.1\%, specificity $99.6 \%$, the accuracy $-98.8 \%$, for CBCT: sensitivity $-97.3 \%$, specificity - 99.1\%, accuracy - 98.4\%, for OPG: sensitivity $78.6 \%$, specificity $-76.3 \%$, accuracy $-77.4 \%$, for radiography of the skull: sensitivity $-61.1 \%$, specificity $-59.2 \%$, accuracy $-60.2 \%$.

Key words: osteonecrosis, multispiral computed tomography, orthopantomography, desomorfin, cone-beam computed tomography, radionuclide diagnostics.

Recommended citation: Babkova A.A., Serova N.S., Medvedev Yu.A., Pasha S.P. Complex radialogical diagnostics in drug-dependent patients with phosphorus osteonecrosis of the facial skeleton at the preoperative stage. Medical Visualization. 2018; 22 (4): 85-95. DOI: 10.24835/1607-0763-2018-4-85-95.

\section{Введение}

Дезоморфин - синтетический дешевый наркотический препарат, изготовляемый кустарным путем благодаря экстрагированию кодеина из кодеинсодержащих препаратов, которые находились в свободной продаже в аптеках на территории России вплоть до 01.06.2012. Также в состав дезоморфина входят различные химические токсичные вещества, такие как бензин, сода, йод, ацетон, различные щелочи и кислоты, красный фосфор и т.д. Вследствие употребления данного наркотического средства у пациентов, преимущественно молодых трудоспособных мужчин, развиваются атипичные остеонекрозы лицевого скелета. Данный вид остеонекроза костей лицевого скелета характеризуется продолжительным течением, склонностью к рецидивам и к развитию осложнений, что в итоге неизбежно приводит к инвалидизации [1-3]. В настоящее время различные группы ученых рассматривают данный тип остеонекроза как вариант ранее описанных атипичных остеомиелитов челюстей, так называемых фосфорных остеонекрозов челюстей, широко встречавшихся в конце XIX - начале XX веков у работников, преимущественно женщин, детей и подростков, многочисленных спичечных фабрик, располагавшихся как на территории стран Европы, так и на территории Российской империи [2-4].

Однако, начиная с 2003 г., на территории РФ и ряда стран СНГ отмечается широкое распространение дезоморфина. Данное наркотическое средство оказалось наиболее популярно среди лиц молодого возраста, преимущественно мужчин. Таким образом, неуклонно увеличивается количество лиц, у которых развивается остеонекроз лицевого (в ряде случаев мозгового) отдела скелета [4-7].

Хроническое внутривенное введение дезоморфина ("крокодил") способствует развитию у наркозависимых лиц атипичных остеонекрозов челюстей и других костей черепа, для которых характерным является затяжное течение, не поддающееся традиционному терапевтическому лечению, а также тяжелых гнойно-воспалительных осложнений с тенденцией к распространению на окружающие ткани $[9,10,13-16]$.

В настоящий момент не существует достоверных данных о возможном распространении дезоморфина за пределы РФ и стран бывшего СССР, в том числе ввиду отсутствия возможности транспортировки и длительного хранения данного наркотического средства [17-20].

Лечение остеонекрозов лицевого скелета в настоящее время заключается в проведении различных оперативных вмешательств в преде- 
лах здоровых тканей с одномоментной или отсроченной установкой никелид-титановых имплантатов на месте резецированной нижней челюсти $[7,10-12,15]$.

Диагностика распространенности остеонекроза и планирование необходимого вида и объема оперативного вмешательства основаны на результатах комплексной лучевой диагностики: ортопантомографии (ОПТГ), рентгенографии (РГ) черепа, мультиспиральной компьютерной томографии (МСКТ), контрастно-лучевой компьютерной томографии (КЛКТ), радионуклидной диагностики (планарной сцинтиграфии, однофотонной эмиссионной компьютерной томографии (ОФЭКТ)). В настоящее время отсутствуют данные о диагностической эффективности различных лучевых методов в обследовании пациентов с фосфорными остеонекрозами лицевого скелета на дооперационном этапе, в том числе КЛКТ. Также практически отсутствуют данные о сопоставимости МСКТ и КЛКТ в диагностике остеонекрозов лицевого скелета и о необходимости применения методов радионуклидной диагностики у дезоморфинзависимых пациентов на дооперационном этапе лечения.

\section{Цель исследования}

Определить роль комплексной лучевой диагностики и диагностическую эффективность различных лучевых методов (РГ, ОПТГ, МСКТ, КЛКТ) у наркозависимых пациентов с фосфорными остеонекрозами лицевого скелета на дооперационном этапе.

\section{Материал и методы}

В период с 2015 по 2018 г. было проведено комплексное клинико-лучевое обследование 85 дезоморфинзависимых пациентов с остеонекрозами лицевого скелета. Возраст больных варьировал от 18 до 48 лет. Средний возраст пациентов составил 27 лет. Преобладали мужчины молодого возраста от 22 до 35 лет ( $n=78 ; 92 \%)$. Все пациенты предъявляли жалобы на обнажение костной ткани челюстей с наличием или без гнойного отделяемого, которое возникало после удаления зубов, появление свищевых ходов различной локализации, неприятного запаха, изменение прикуса, конфигурации лица, затруднение нормального акта жевания. Комплексная лучевая диагностика включила в себя проведение ОПТГ, РГ черепа, МСКТ, КЛКТ у 85 (100\%) пациентов, радионуклидной диагностики (планарной остеосцинтиграфии, ОФЭКТ) у 19 (22,4\%) пациентов. Далее все пациенты были направлены на проведение хирургического лечения в объеме различных резекций костей лицевого скелета.
По данным гистологического исследования фрагментов удаленных костных и мягкотканных фрагментов у всех пациентов подтверждался диагноз "токсический фосфорный остеонекроз" соответствующих костей лицевого скелета.

РГ черепа проводилась на аппарате Silhouette HF General Electric Medical Systems, ОПТГ - на аппарате Orthopantograph OP 100, МСКТ - на мультиспиральном компьютерном томографе Somatom Sensation-40 (Siemens, Германия), а также Toshiba Aquilion ONE 640 (Toshiba, Япония). При проведении данного исследования у всех пациентов (n = 85; 100\%) использовались мультипланарные и 3D-реконструкции полученных диагностических данных с последующим анализом. КЛКТ у пациентов с остеонекрозами лицевого скелета на дооперационном этапе лечения проводилась на аппарате Galileos Comfort (Sirona, Германия) с применением специального режима FaceScanner, при котором происходит наложение фотографического изображения лица пациента на 3D-peконструкцию его черепа.

На дооперационном этапе лечения дезоморфинзависимых пациентов с остеонекрозами лицевого скелета по данным комплексной лучевой диагностики (ОПТГ, РГ черепа, МСКТ, КЛКТ) оценивали следующие параметры: локализация очагов остеонекроза (в области челюстных костей), возможное распространение патологического процесса на другие кости лицевого скелета и мозгового отдела черепа (стенок глазниц, скуловых костей, отделов крыловидной кости, сошника, небной кости и т.д.), изменение окружающей очаг остеонекроза костной ткани, наличие перелома нижней челюсти, локализация и особенности периостальных наслоений, состояние височнонижнечелюстных суставов, оценка секвестрации, вовлеченность в патологический процесс каналов нижнечелюстных нервов, изменения характера периодонтальной щели сохранных зубов, оценка лунок удаленных зубов, состояние околоносовых синусов, полости носа, окружающих мягких тканей (наличие отека, свищевых ходов).

19 (22\%) пациентам также была проведена радионуклидная диагностика: планарная остеосцинтиграфия и ОФЭКТ на гамма-камере Millennium MG (General Electric, США) с применением радиофармпрепарата (РФП) 99mТс-пирфотеха по стандартной методике. В результате проведенного сцинтиграфического исследования получали серию томографических срезов области исследования путем реконструкции с использованием алгоритма OSEM/MLEM.

Радионуклидная диагностика у дезоморфинзависимых пациентов с остеонекрозами лицевого 
скелета на дооперационном этапе проводилась 19 пациентам при подозрении на распространение патологического процесса за пределы лицевого скелета: по данным осмотра, жалоб, отсутствия травм, заболеваний костно-суставной системы в анамнезе. Оценка степени гиперфиксации РФП производилась визуально в сравнении с контралатеральными или ниже-/вышележащими референсными зонами.

Результаты лучевых обследований (ОПТГ, РГ, МСКТ, КЛКТ) у пациентов с остеонекрозами лицевого скелета вводились в специальную таблицу в программе Numbers (2.0). Введенная информация обрабатывалась методом вариационной статистики с помощью компьютерной программы Numbers (2.0). Диагностическая эффективность различных методов лучевой диагностики в представленном исследовании изучалась на основании определения: чувствительности, точности, специфичности.

Верификация полученных результатов проведенных исследований осуществлялась по данным гистологического исследования материала, взятого во время оперативного вмешательства из краевых отделов участков резекции.

\section{Результаты}

По данным комплексного лучевого обследования на предоперационном этапе в зависимости от локализации остеонекрозов лицевого скелета все пациенты ( $\mathrm{n}=85 ; 100 \%$ ) были распределены следующим образом: в 48\% (n = 41) случаев зона остеонекроза локализовалась в области нижней челюсти, в 24\% (n = 20) - в области верхней челюсти, в 28\% ( $n=24)$ патологический процесс захватывал обе челюсти.

Данные МСКТ и КЛКТ позволили выявить распространение патологического процесса на различные кости лицевого отдела скелета: у 35\% пациентов ( $\mathrm{n}=30$ ) определялось вовлечение в патологический процесс стенок верхнечелюстных синусов, скуловых, небных отростков ( $n=35 ; 41 \%$ ), лобных отростков верхнечелюстных костей, небной кости, различных стенок глазниц ( $n=7 ; 8 \%)$. В 29,5\% случаев ( $n=25)$ отмечалось распространение патологического процесса на глазничную часть лобной кости, клиновидную кость, сошник (рис. 1).

По данным РГ черепа, ОПТГ, МСКТ и КЛКТ у пациентов с обширным поражением нижней челюсти был выявлен патологический перелом (n = 19; 22,3\%) (рис. 2).

В представленном исследовании периостальная реакция определялась у 50 (59\%) пациентов. Преобладающим типом ( $n=23 ; 46 \%)$ явился так называемый муфтообразный слоистый, местами разорванный периостит, локализующийся в области нижней челюсти (рис. 3).

Точную локализацию и характер секвестров по данным РГ черепа и ОПТГ определить не представлялось возможным ввиду наличия у данных методов проекционных искажений. Однако МСКТ и КЛКТ позволили выявить у 35 (41\%) пациентов секвестры различного, преимущественно смешанного характера как в области челюстей, так и других костей черепа.

Как классические рентгенологические методы (РГ, ОПТГ), так и МСКТ, КЛКТ позволили определить изменения периодонтальных щелей сохранных зубов - склероз и расширение у 35 (41\%) пациентов. Лунки удаленных зубов не визуализировались в $66 \%$ случаев ( $\mathrm{n}=56)$, в $34 \%$ случаев ( $n=29$ ) отмечался склероз лунок удаленных зубов без признаков положительного ремоделирования.

По данным ОПТГ, РГ черепа, МСКТ и КЛКТ остеосклероз нижней челюсти и/или верхней челюсти определялся у 85 (100\%) пациентов (рис. 4). Однако МСКТ (КЛКТ) позволила оценить точную локализацию и протяженность зон остеосклероза не только в челюстях, но и в других костях лицевого (мозгового) скелета. Очаги остеосклероза помимо челюстей определялись в различных отделах клиновидной кости, переднем скате затылочной кости, скуловых и височных костях.

Ввиду ограниченности ОПТГ и РГ черепа достоверная оценка состояния околоносовых синусов и полости носа оказалась невозможной, тогда как по данным МСКТ (КЛКТ) вовлечение в патологический процесс околоносовых синусов было выявлено у 30 (35\%) пациентов, структур полости носа - у 12 (14,1\%) пациентов (рис. 5).

Также по данным РГ черепа и ОПТГ выявление степени вовлеченности каналов нижнечелюстных нервов в патологический процесс при обширном поражении нижней челюсти оказалось невозможным, однако МСКТ (КЛКТ) позволили выявить деструкцию стенок каналов нижнечелюстных нервов в 27 (31,8\%) случаях.

Изменения со стороны окружающих мягких тканей в виде асимметрии, отека были отмечены у $70(82,3 \%)$ пациентов, у 45 (53\%) пациентов определялись единичные или множественные свищевые ходы различной локализации. Однако данная оценка оказалась возможной только при применении МСКТ, так как ОПТГ, РГ черепа и КЛКТ имеют ограничения в визуализации мягких тканей.

19 (22\%) пациентам на дооперационном этапе также была проведена радионуклидная диагностика, включившая в себя планарную остеосцинтиграфию и ОФЭКТ. Критериями оценки получен- 

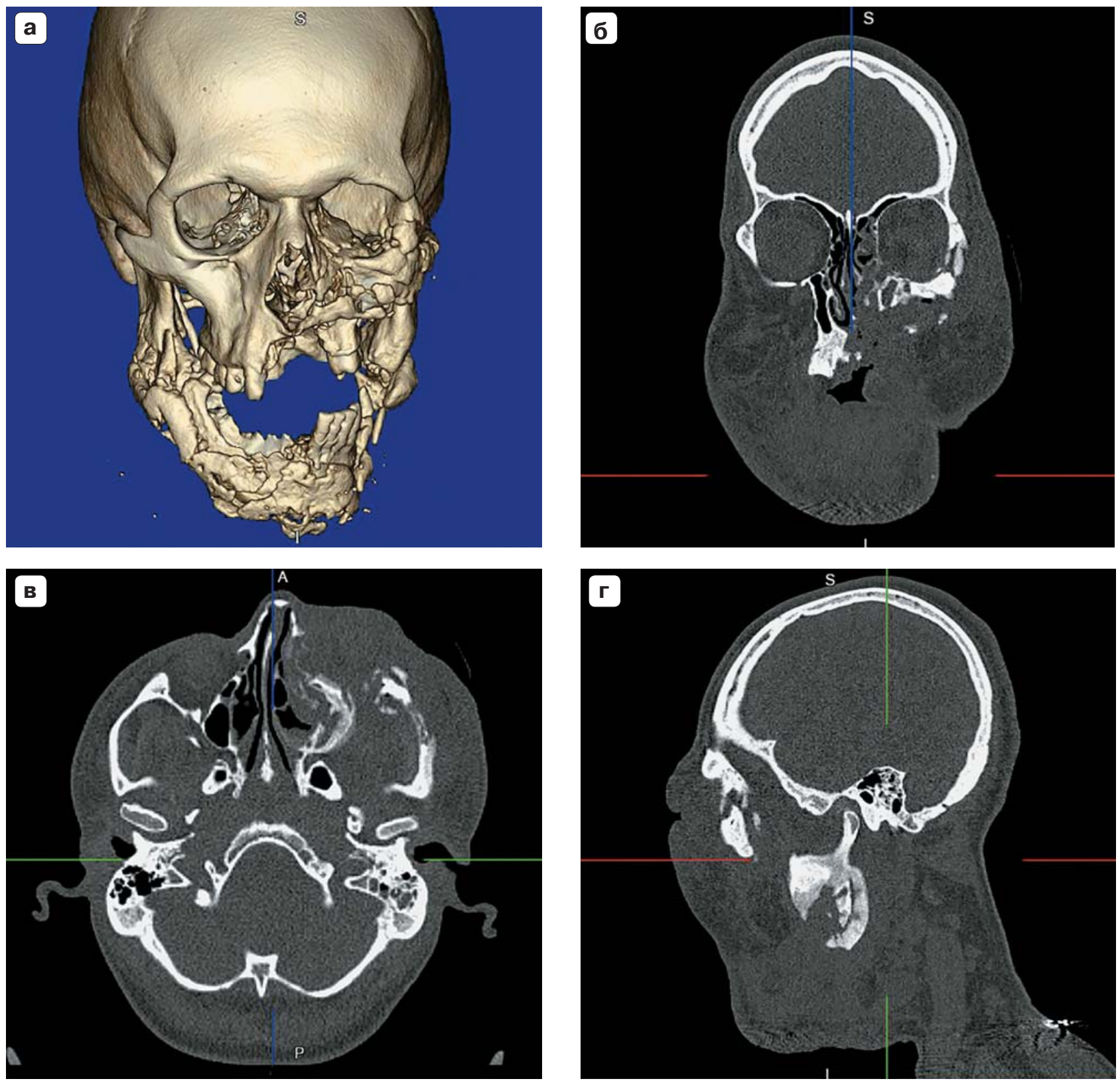

Рис. 1. MCKT. 3D (а), корональная (б), аксиальная (в), сагиттальная (г) реконструкции. Отмечаются костно-деструктивные изменения в области альвеолярного и небного отростка верхней челюсти слева с распространением процесса на стенки левого верхнечелюстного синуса, левые отделы носовой полости, нижнюю и латеральную стенки левой орбиты, левую скуловую кость, скуловой отросток левой височной кости, с наличием периостальных наслоений, развитием секвестрации. 


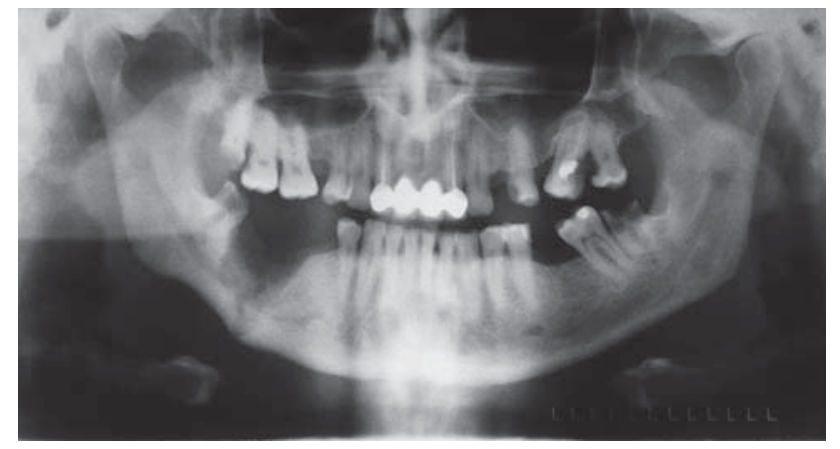

Рис. 2. Ортопантомограмма. Определяется остеосклероз нижней челюсти справа. В области 4.5-4.6 отмечается участок деструкции костной ткани с неровными и местами нечеткими контурами. В области участка деструкции визуализируется линия перелома без смещения костных отломков.
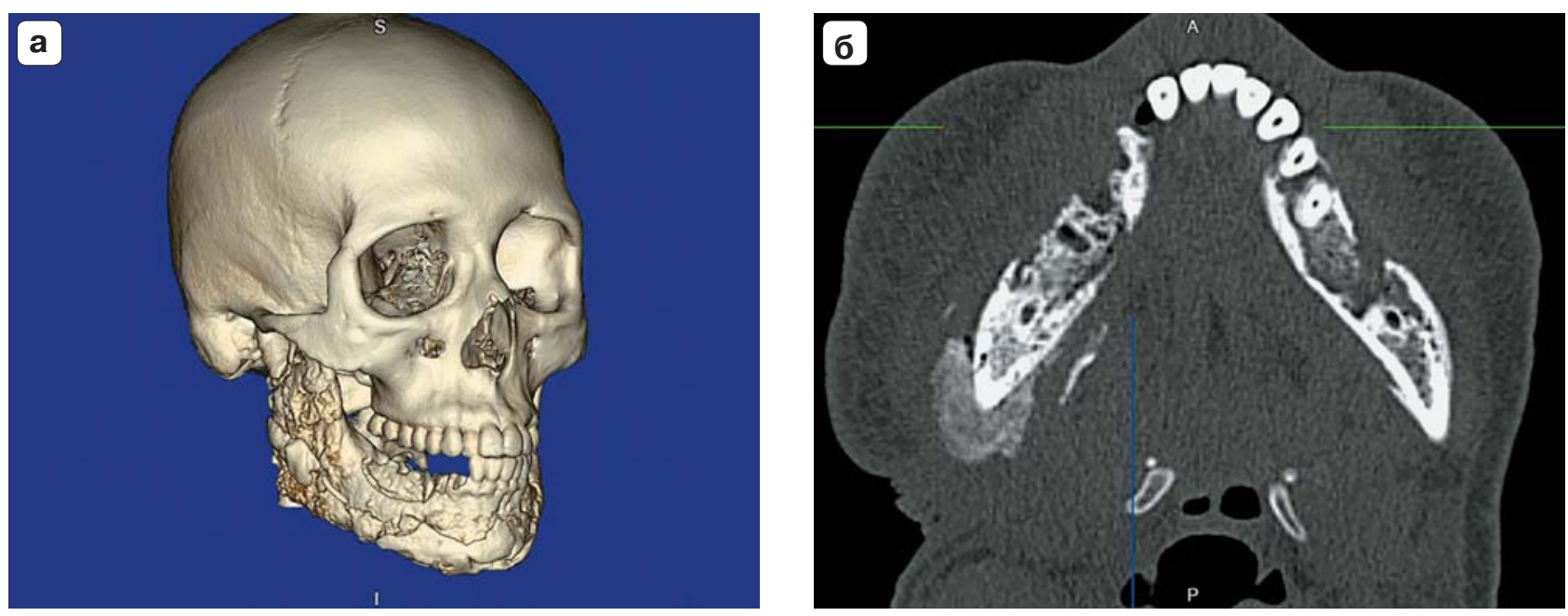

Рис. 3. МСKT. 3D (а), аксиальная (б) реконструкции. В области нижней челюсти отмечается неравномерный остеосклероз. В области тела, угла, ветви, отростков нижней челюсти справа визуализируются массивные бахромчатые, местами разорванные периостальные наслоения.

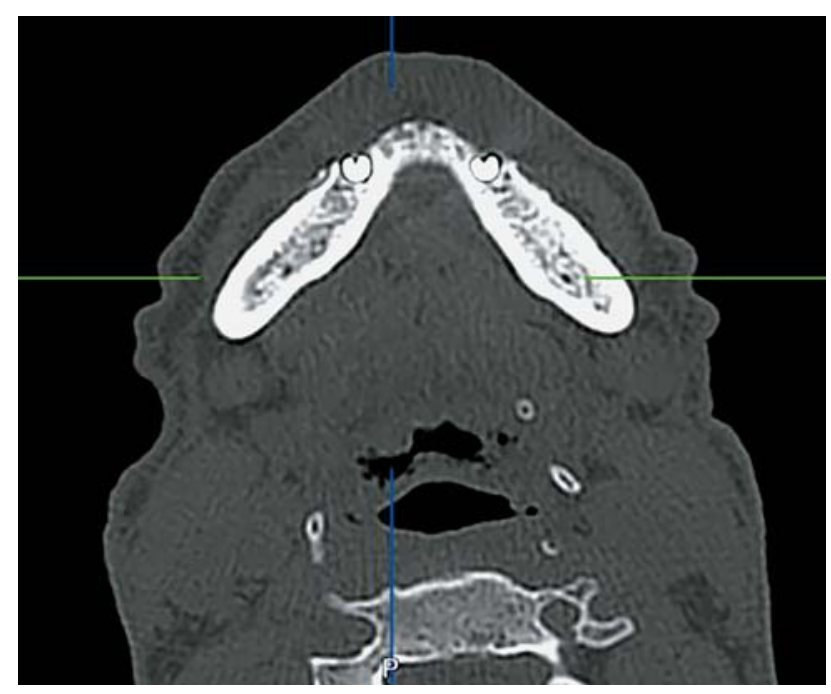

Рис. 4. МСКТ. Аксиальная реконструкция. Отмечается тотальный остеосклероз нижней челюсти. 

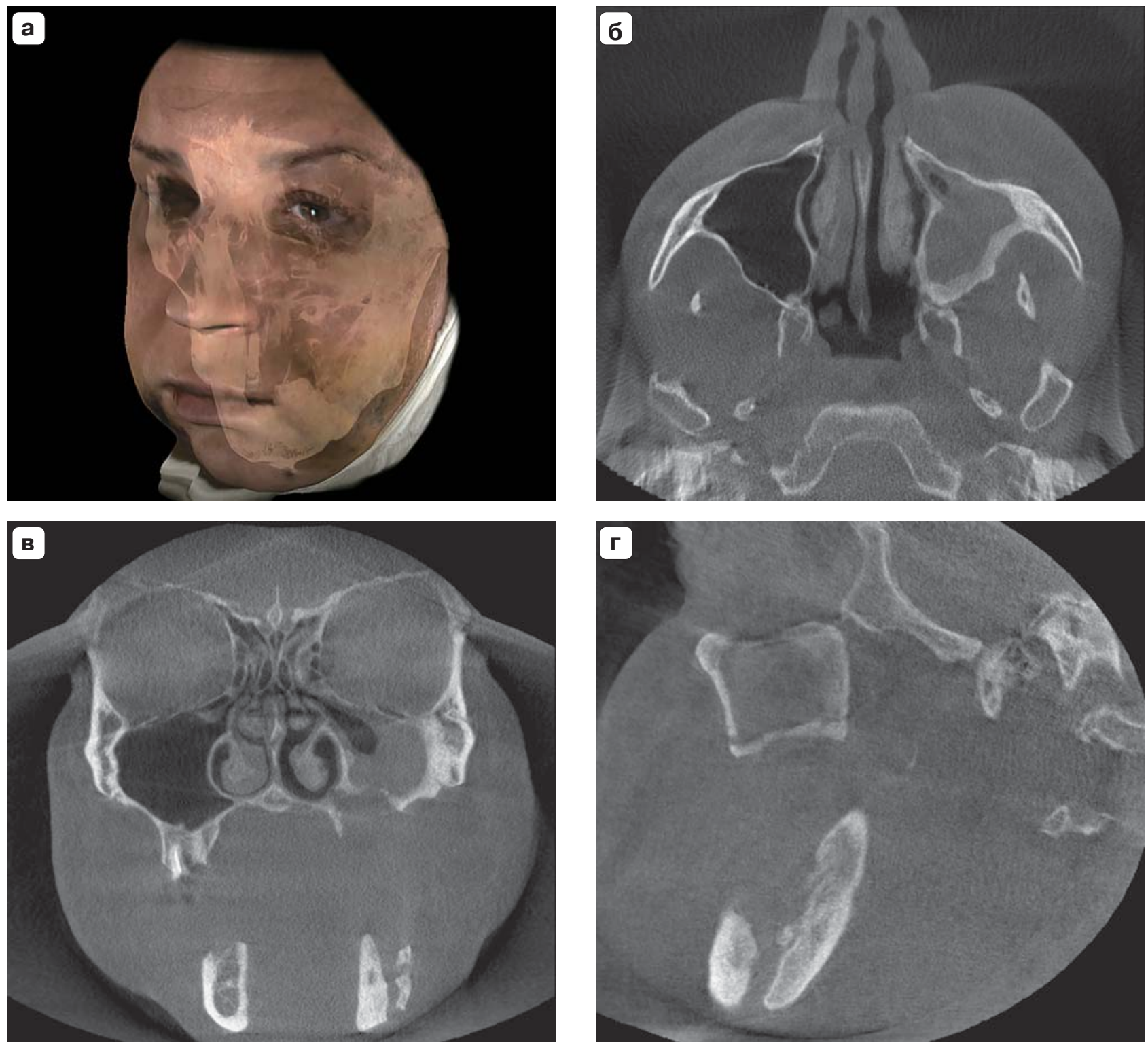

Рис. 5. КЛКТ. 3D (а), аксиальная (б), корональная (в), сагиттальная (г) реконструкции. Визуализируются деструктивные изменения альвеолярного и небного отростков верхней челюсти слева с формированием ороантрального соустья, склероз и утолщение стенок левого верхнечелюстного синуса. Левый верхнечелюстной синус субтотально заполнен содержимым. Отмечаются деформация и остеосклероз нижней челюсти. В области тела нижней челюсти слева определяется линия перелома со смещением костных отломков. Краевые отделы в области перелома четкие, неровные, склерозированные.

ных диагностических данных явились локализация и степень гиперфиксации РФП.

При проведении радионуклидной диагностики у всех пациентов определялась гиперфиксация РФП в области верхней, нижней челюстей ( $\mathrm{n}=19$, $100 \%)$, а также в области стенок глазниц - у 6 (32\%) пациентов, скуловых костей ( $n=5 ; 26 \%)$. При этом в ряде случаев накопление РФП также отмечалось за пределами лицевого скелета. В представленном исследовании гиперфиксация РФП наблюда- лась в проекции тела, рукоятки грудины (n = 6; $32 \%)$, в области головок плечевых костей ( $n=4$; $21 \%)$, в проекции гребней подвздошных костей (n = 5; 26\%), у 2 (11\%) пациентов - в проекции коленного сустава (рис. 6, 7).

Таким образом, радионуклидное исследование позволило дополнить результаты ОПТГ, РГ черепа, МСКТ, КЛКТ и предположить наличие остеонекротических изменений за пределами костей черепа. 


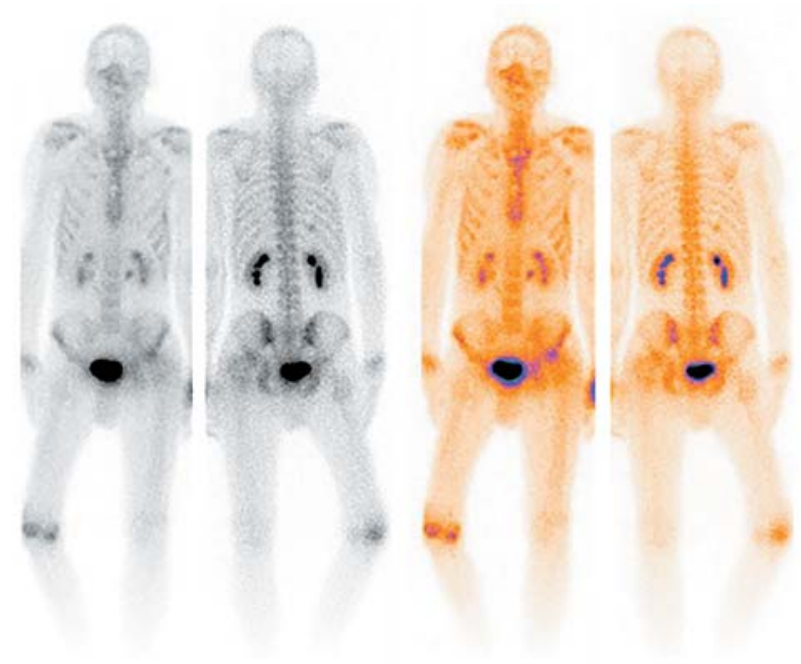

Рис. 6. Остеосцинтиграмма. Определяются зоны повышенного накопления РФП в проекции тела верхней челюсти и скулоглазничного комплекса справа, полости носа, в области правого коленного сустава, рукоятки и тела грудины.

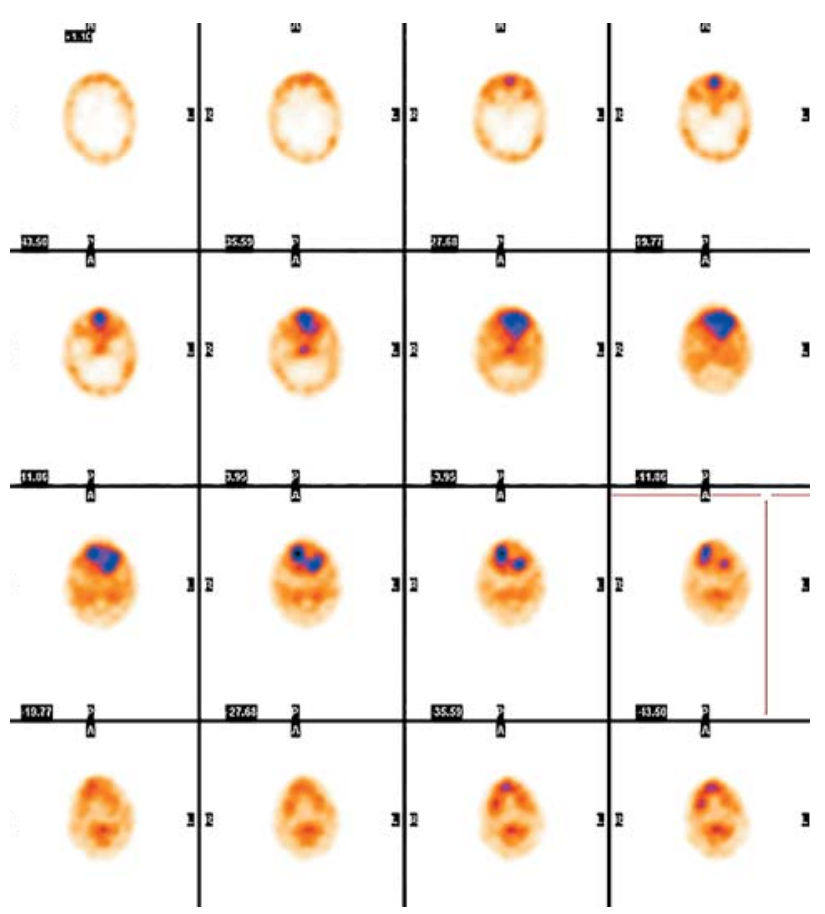

Рис. 7. ОФЭКТ, аксиальные реконструкции. Отмечается гиперфиксация РФП в области носовой полости, скулоглазничного комплекса слева, верхней челюсти, нижней челюсти.

\section{Обсуждение}

Несмотря на введенный на законодательном уровне в 2012 г. запрет на безрецептурную продажу кодеинсодержащих лекарственных препаратов с целью борьбы со вспышкой наркомании, ежедневно в ведущие больницы Российской Федерации обращаются десятки пациентов, употребляющих дезоморфин. Таким образом, остеонекрозы лицевого скелета у дезоморфинзависимых пациентов являются острой и актуальной социально-медицинской проблемой. При этом развитие данного заболевания у преимущественно мужчин молодого возраста приводит к дальнейшей инвалидизации и социальной дезадаптации.

В настоящее время отсутствуют единые протоколы и алгоритмы лучевого обследования данной категории пациентов. Комплексная лучевая диагностика играет ведущую роль в оценке локализации, распространенности очагов остеонекроза, что является необходимыми данными для планирования оптимального последующего хирургического лечения.

В связи с наличием существенных недостатков классических рентгенологических методов (РГ черепа, ОПТГ), таких как получение однопроекционного, плоскостного изображения, наличие суммационного эффекта и недостаточная визуализация фронтальных отделов челюстей, околоносовых синусов, полости носа, невозможность оценки истинной распространенности патологического процесса за пределами челюстей и в окружающих мягких тканях. Традиционные рентгенологические методики (ОПТГ, РГ черепа) позволили составить лишь ориентировочное, неполное представление о распространенности и характере патологических изменений. Данные этих методик значительно уступают в показателях диагностической эффективности таким высокоинформативным методам, как МСКТ и КЛКТ, и не могут применяться для планирования дальнейшего хирургического лечения, что совпадает с данными отечественных авторов $[5,6,9]$.

Высокотехнологичные и информативные методы лучевой диагностики (МСКТ, КЛКТ) позволяют своевременно получить необходимый объем диагностической информации у пациентов с остеонекрозами лицевого скелета на дооперационном этапе. Данные методы позволяют выявить локализацию патологического процесса, изменения окружающих мягких тканей. Диагностические возможности МСКТ и КЛКТ у пациентов с остеонекрозами лицевого скелета на фоне дезоморфиновой зависимости оказались практически полностью сопоставимы. КЛКТ позволила корректно выявить особенности патологических изменений у пациен- 


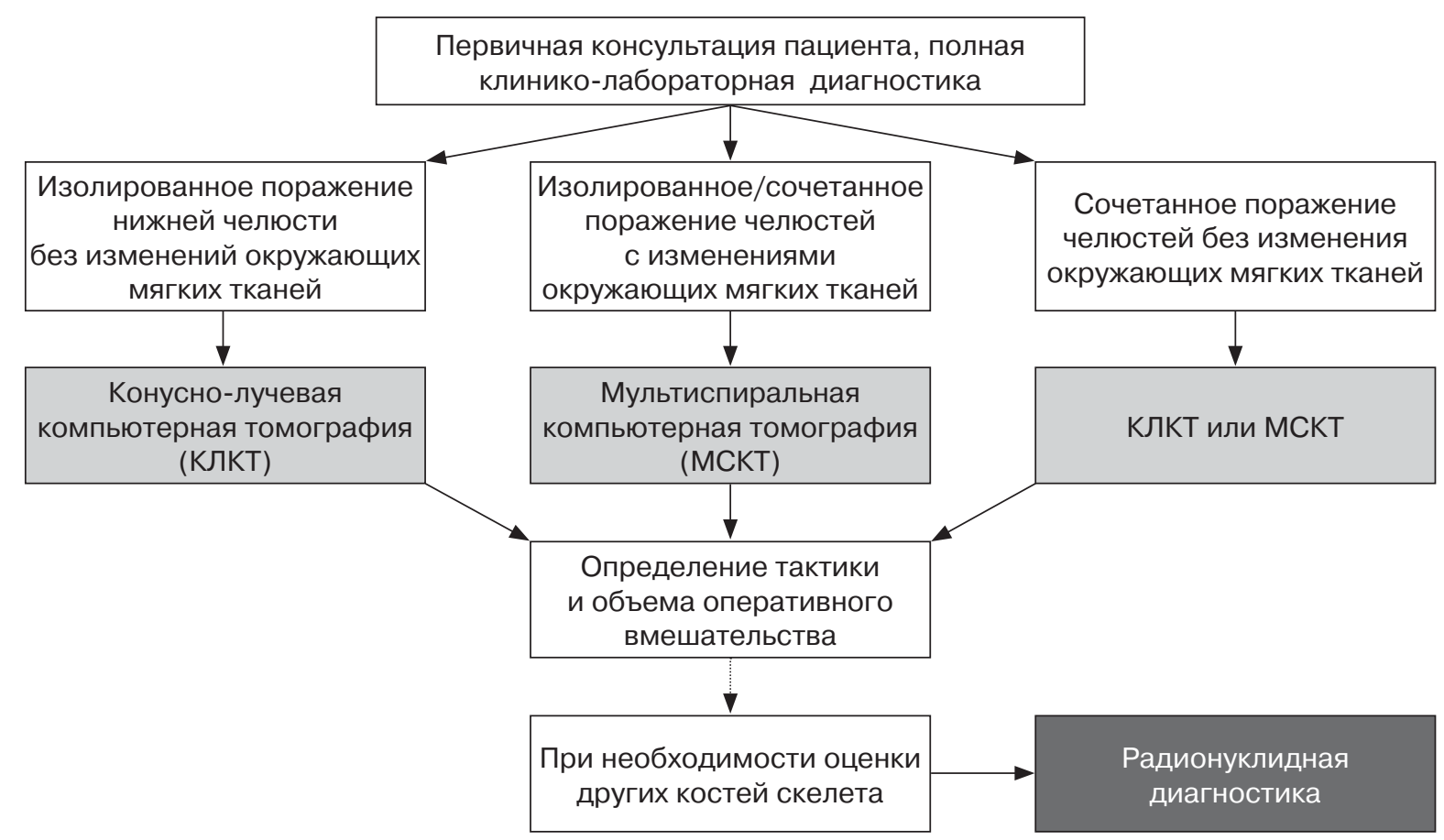

Рис. 8. Схема алгоритма лучевого обследования пациентов с остеонекрозами лицевого скелета на дооперационном этапе лечения.

тов с остенекрозами костей лицевого скелета, в том числе определить характер и локализацию изменений костей мозгового отдела черепа. Недостатком метода явилось отсутствие полноценной и корректной визуализации окружающих мягких тканей, при выявлении свищевых ходов.

При этом данные о возможностях и информативности КЛКТ у пациентов с остеонекрозами костей лицевого скелета как в отечественной, так и в зарубежной литературе на настоящий момент отсутствуют, что дало основание для изучения показателей диагностической эффективности КЛКТ в представленном научном исследовании.

Также в настоящее время в литературе практически отсутствуют данные о применении радионуклидной диагностики у пациентов с остеонекрозами лицевого скелета и гистологической верификации выявленных изменений в костях за пределами черепа [13]. Таким образом, данный вопрос требует дальнейшего всестороннего изучения.

На дооперационном этапе лечения пациентов с остеонекрозами костей лицевого скелета на фоне употребления дезоморфина МСКТ превзошла КЛКТ незначительно, при меньшей у КЛКТ лучевой нагрузке, тогда как оба этих метода значительно превзошли традиционные рентгенологические методы (ОПТГ, РГ черепа) по всем показателям диагностической эффективности. Таким образом, исходя из данных, представленных выше, оказалось возможным составить алгоритм оптимального лучевого обследования пациентов с остеонекрозами костей лицевого скелета на дооперационном этапе лечения (рис. 8).

\section{Заключение}

Комплекс современных высокоинформативных методов (МСКТ, КЛКТ, радионуклидная диагностика) является неотъемлемым этапом обследования дезоморфинзависимых пациентов с остеонекрозами лицевого скелета на дооперационном этапе.

Для верификации показателей диагностической эффективности методов лучевой диагностики, используемых в представленном исследовании, применялись данные гистологического исследования биоптата. Биопсию выполнили во время оперативного вмешательства из участков визуально пораженной и визуально неизмененной костной ткани в нескольких точках. Данные исследования биоптата явились критерием для установления наличия или отсутствия остеонекротического процесса в костных структурах области исследования. Во всех случаях (n = 85; 100\%) зоны, определяемые по данным комплексного лучевого обследования как области патологических изменений, совпали с данными исследования биоптатов, в которых гистологически был верифицирован остеонекроз. 
По данным проведенного исследования показатели диагностической эффективности для МСКТ составили: чувствительность - 98,1\%, специфичность - 99,6\%, точность - 98,8\%, для КЛКТ: чувствительность - 97,3\%, специфичность - 99,1\%, точность - 98,4\%, для ОПТГ: чувствительность $78,6 \%$, специфичность - 76,3\%, точность - 77,4\%, для РГ черепа: чувствительность - 61,1\%, специфичность - 59,2\%, точность - 60,2\%.

\section{Список литературы}

1. Маланчук В.О., Копчак А.В., Бродецький І.С. Клінічні особливості остеомієліту щелеп у хворих з наркотичною залежністю. Укр. мед. часопис. 2007; 4 (60): 111-117.

2. Баранник Н.Г., Варжапетян С.Д., Мосейко А.А., Манухина О.Н. Опыт лечения пациентов с остеомиелитом челюстей и вторичным иммунодефицитом на фоне приёма наркотических препаратов. Актуальні питання медичної науки та практики. 2013; 1 (80): 12-20.

3. Маланчук В.О., Бродецький І.С., Забудська Л.Р. Особливості рентгенологічної картини остеомієліту щелеп у хворих на фоні наркотичної залежності. Укр. мед. часопис. 2009; 2 (70): 122-125.

4. Серова Н.С., Курешова Д.Н., Бабкова А.А., Басин Е.М. Многосрезовая компьютерная томография в диагностике токсических фосфорных некрозов челюстей. Вестник рентгенологии и радиологии. 2015; 5: 11-16.

5. Иващенко А.Л., Матрос-Таранец И.Н., Прилуцкий А.С. Современные аспекты этиопатогенеза, клинической картины и лечения остемиелитов челюстей у пациентов с наркотической зависимостью и ВИЧ-инфекцией. Збірник статей. 2009: 1 (13): 213-219.

6. Маланчук В. А., Бродецкий И.С. Комплексное лечение больных остеомиелитом челюстей на фоне наркотической зависимости. Вестник ВГМУ. 2014; 2 (13): $115-123$.

7. Бабкова А.А., Курешова Д.Н., Серова Н.С., Паша С.П., Басин Е.М. Комплексная лучевая диагностика остеонекрозов у дэезоморфинзависимых пациентов. REJR. 2015; 5 (4): 13-23.

8. Серова Н.С. Лучевая диагностика в стоматологической имплантологии: Дис. ... д-ра мед. наук. М., 2010. 168 c.

9. Медведев Ю.А, Басин Е.М. Фосфорные некрозы челюстей. Врач. 2012; 1: 21-25.

10. Тимофеев А.А., Дакал А.В. Клиническое течение гнойно-воспалительных заболеваний челюстей и мягких тканей челюстно-лицевой области у больных, употребляющих наркотик "винт". Современная стоматология. 2010; 1: 96-102.

11. Погосян Ю.М., Акопян К.А., Гаспарян Л.Л. Рентгенодиагностика остеонекроза челюстей у больных, употребляющих наркотическое средство "крокодил". Вопросы теоретической и клинической медицины. 2013; 2 (78): 44-49.

12. Рузин Г.П., Ткаченко О.В. Клинические проявления токсического остемиелита в зависимости от давности употребления наркотика. Український стоматологічний альманах. 2015; 1: 47-52.

13. Бабкова А.А., Паша С.П., Серова Н.С. Возможности применения методов радионуклидной диагностики у пациентов с дезоморфиновой зависимостью. REJR. 2017; 7 (3): 136-143.

14. Национальное руководство / Главный редактор тома С.К. Терновой. М.: ГЭОТАР-Медиа, 2013. Сер. Национальные руководства по лучевой диагностике и терапии / Главный редактор серии С.К. Терновой. 1000 с.

15. Серова Н.С. Дентальная объемная томография в решении некоторых задач стоматологии и челюстно-лицевой хирургии. Эдодонтия TODAY. 2010; 2: 55-57.

16. Серова Н.С., Курешова Д.Н., Бабкова А.А., Басин Е.М. Рентгенологическая классификация остеонекрозов средней зоны лица у пациентов с наркотической зависимостью от дезоморфина и первитина. Диагностическая и интервенционная радиология. 2016; 10 (3): 35-41.

17. Thekkemuriyi D.V., John S.G., Pillai U. 'Krokodil'a designer drug from across the Atlantic, with serious consequences. Am. J. Med. 2014: 127.

18. Mullins M.E., Schwarz E.S. "Krokodil" in the United States Is an Urban Legend and Not a Medical Fact. Am. J. Med. 2014; 127 (7): 25.

19. No Confirmed Reports of Desomorphine ("Krokodil"/ "Crocodile") in Canada (PDF). Canadian Centre on Substance Abuse. November 21, 2013.

20. Skowronek R., Celiński R., Chowaniec C. "Crocodile" new dangerous designer drug of abuse from the East. Clin. Toxicol. 2012; 50 (4): 269.

\section{References}

1 Malanchuk V.A., Kopchak A.V., Brodetsky I.S. Clinical features of jaw osteomyelitis in drug addict patients. Ukr. med. chasopis. 2007; 4 (60): 111-117. (In Ukrainian)

2 Barannik N.G., Varzhapetyan S.D., Moseiko A.A., Manuchina O.N. The experience of treatment of patients with osteomyelitis of the jaws and secondary immunodeficiency on a background of narcotic drugs. Aktual"ni pitannja medichnoï nauki ta praktiki. 2013; 1 (80): 12-20. (In Russian)

3 Malanchuk V.O., Brodetsky I.S., Zabudska L.R. Radiological features of the jaws osteomyelitis in drugaddicted patients. Ukr. Med. Chasopis. 2009; 2 (70): 122-125. (In Ukrainian)

4 Serova N.S., Kureshova D.N., Babkova A.A., Basin E.M. Multislice computed tomography in the diagnosis of toxic phosphorus necrosis of the jaw. Vestnik rentgenologii i radiologii. 2015; 5: 11-16. (In Russian)

5 Ivashchenko A.L., Matros-Taranets I.N., Prilutskiy A.S. Modern aspects of the etiopathogenesis, clinical presentation and treatment of osteomyelitis of jaws at patients with narcotic dependence and a HIV-infection. Zbirnik statej. 2009: 1 (13): 213-219. (In Russian)

6 Malanchuk V.O., Brodetsky I.S. Complex treatment of patients with osteomyelitis of the jaws against the background of drug addiction. Vestnik Vgmu. 2014; 2 (13): 115-123. (In Russian)

7 Babkova A.A., Kureshova D.N., Serova N.S., Pasha S.P., Basin E.M. Complex radiological diagnosis of osteonecrosis in desomorphine dependent patients. REJR. 2015; 5 (4): 13-23. (In Russian)

8 Serova N.S. Radiology in stomatology and implantology: Doct. dis., M., 2010. 168 p. (In Russian)

9 Medvedev Yu.A., Basin E.M. Phosphorus necrosis of the jaws. Doctor. 2012; 1: 21-25. (In Russian) 
10 Tymofieiev A., Dakal A. Clinical course of purulent inflammatory deseases of the jaws and soft tissues of the maxillofacial area in patients using "vint" drug. Modern dentistry. 2010; 1: 96-102. (In Russian)

11 Poghosyan Yu.M., Hakobyan K.A., Gasparyan L.L. Radiographic diagnosis of jaw osteonecrosis at patients who use the drug "Krokodil". Issues of clinical and theoretical medicine. 2013; 2 (78): 44-49. (In Russian)

12 Ruzin G.P., Tkachenko O.V. Clinical manifestations of toxic osteomielita depending on prescription drug. Ukrainian dental almanac. 2015; 1: 47-52. (In Russian)

13 Babkova A.A., Pasha S.P., Serova N.S. Possibilities of application of methods of radionuclide diagnostics in patients with desomorphine dependence. REJR. 2017; 7 (3): 136-143. (In Russian)

14 National guide / Volume ed. S.K. Ternovoy. Moscow: GEOTAR-Media, 2013. National guides in radiation diagnosis and therapy. Ed. in Chief S. K. Ternovoy. 1000 p. (In Russian)
15 Serova N.S. Dental volumetric tomography in the solution of some problems of dentistry and maxillofacial surgery. Endodontia TODAY. 2010; 2: 55-57. (In Russian)

16 Kureshova D.N., Serova N.S., Babkova A.A., Basin E.M. X-Ray classification of osteonecrosis of the midface in patients with drug-addiction from desomorphine and pervitin. Diagnostic and interventional radiology. 2016; 10 (3): 35-41. (In Russian)

17 Thekkemuriyi D.V., John S.G., Pillai U. 'Krokodil'a designer drug from across the Atlantic, with serious consequences. Am. J. Med. 2014; 127.

18 Mullins M.E., Schwarz E.S. 'Krokodil' in the United States Is an Urban Legend and Not a Medical Fact. Am. J. Med. 2014; 127 (7): 25

19 No Confirmed Reports of Desomorphine ("Krokodil"/ "Crocodile") in Canada (PDF). Canadian Centre on Substance Abuse. November 21, 2013.

20 Skowronek R., Celiński R., Chowaniec C. "Crocodile" new dangerous designer drug of abuse from the East. Clin. Toxicol. 2012; 50 (4): 269.

Для корреспонденции*: Бабкова Анна Анатольевна - 127473 Москва, Селезневская ул., д. 30, корп. 3, кв. 18. Тел.: + 7-903-662-55-73. E-mail: an4i1@yandex.ru

Бабкова Анна Анатольевна - ассистент кафедры лучевой диагностики и лучевой терапии лечебного факультета ФГАОУ ВО Первый МГМУ им. И.М. Сеченова, врач-рентгенолог кабинета рентгеновской компьютерной томографии и маммографии УКБ №1, Москва.

Серова Наталья Сергеевна - член-корр. РАН, доктор мед. наук, профессор кафедры лучевой диагностики и лучевой терапии лечебного факультета ФГАОУ ВО Первый МГМУ им. И.М. Сеченова, врач-рентгенолог кабинета рентгеновской компьютерной томографии и маммографии УКБ №1, Москва.

Медведев Юрий Алексеевич - доктор мед. наук, профессор, заведующий кафедрой челюстно-лицевой хирургии ФПДО ФГБОУ ВО МГМСУ им. А.И. Евдокимова Минздрава России, Москва.

Паша Сергей Платонович - канд. мед. наук, заведующий радионуклидным диагностическим отделением УКБ №1, Москва.

Contact*: Anna A. Babkova - 127473 Moscow, Seleznevskaya str., 30, korp. 3, apt. 18. Phone: + 7-903-662-55-73. E-mail: an4i1@yandex.ru

Anna A. Babkova - assistant of the Department, Department of Radiology, radiologist of the cabinet of x-ray computed tomography and mammography of the University clinical hospital № 1, Moscow.

Natalya S. Serova - corresponding member of the Russian Academy of Sciences, doct. of med. sci., Professor, Department of radiology, Sechenov University, radiologist of the cabinet of x-ray computed tomography and mammography of the University clinical hospital № 1, Moscow.

Yuriy A. Medvedev - doct. of med. sci., Professor, Chief of the Department of Maxillofacial Surgery of A.I. Yevdokimov Moscow State University of Medicine and Dentistry, Moscow.

Sergey P. Pasha - cand. of med. sci., Chief of the radionuclide diagnostic Department of the University clinical hospital № 1, Moscow. 